Análisis. Revista de investigación filosófica, Vol. 1, nº 1, 2014, pp. 181-212

\title{
EL PROBLEMA DEL FISICALISMO NO EXISTE*
}

\author{
Tim Crane y David Hugh Mellor
}

\section{1. ¿QUÉ ES EL FISICALISMO?}

A muchos filósofos les impresiona el progreso logrado por las ciencias físicas. Esto ha tenido un efecto especialmente profundo en sus concepciones ontológicas: ha convertido a muchos de ellos en fisicalistas. Los fisicalistas creen que todo es físico: más precisamente, que todas las entidades, propiedades, relaciones, y hechos son los que estudia la física u otras ciencias físicas. Quizás no todos estén de acuerdo con el espíritu de la conocida observación de Rutherford, según la cual "podemos hacer Física, o bien podemos coleccionar sellos", pero todos conceden a la ciencia física una autoridad ontológica única: la autoridad de decirnos lo que existe.

Actualmente el fisicalismo es casi la ortodoxia para buena parte de la filosofía, y notoriamente para buena parte de la reciente filosofía de la mente. Pero aunque se lo invoca a menudo, rara vez se lo define explícitamente. Habría que definirlo. La pretensión de que todo es físico no es tan clara como parece. En este artículo examinamos algunas de las definiciones del fisicalismo que se han propuesto y algunas razones para ser fisicalistas. Argumentaremos que el fisicalismo carece de una definición clara y creíble, y que no es verdadero en ninguna interpretación que no sea vacía.

En este artículo sólo nos ocuparemos de fisicalismo en tanto que doctrina acerca del mundo empírico. En particular, no hay que confundirlo con el nominalismo, la doctrina de que no existen los universales. ${ }^{2}$ El nominalismo y el fisicalismo son doctrinas completamente independientes. Quienes creen en los universales pueden tanto afirmar como negar consistentemente que las

\footnotetext{
* Traducción de TIM CRANE and D. H. MELLOR, "There is No Question of Physicalism" Mind (1990) XCIX (394): 185-206 doi:10.1093/mind/XCIX.394.185. Disponible en: http://mind.oxfordjournals.org/content/XCIX/394/185.full.pdf. Traducción de José Luis López de Lizaga.

${ }^{1}$ Longuet-Higgins (1972, p. 16).

${ }^{2}$ Contra lo que afirman H. H. Field (1980), y B. Stroud (1987, p. 264).
} 
únicas propiedades y relaciones son las que estudia la ciencia física. Y con la misma consistencia, los nominalistas pueden afirmar o negar que la ciencia física podría proporcionar predicados suficientes para describir el mundo. Esta es la cuestión que importa a los fisicalistas, no si los predicados físicos nombran universales reales. (Por razones de brevedad, escribiremos como si lo hicieran, pero no necesitamos ese supuesto.)

Por consiguiente, tal como lo interpretaremos aquí, el fisicalismo no es una doctrina acerca de los universales u otros objetos abstractos, sino acerca del mundo empírico, y específicamente acerca de las mentes. Afirma que las entidades, propiedades, relaciones y hechos mentales son en realidad físicos. Lo mental es el principal objetivo de los ataques del fisicalismo, pero nosotros pensamos que es un objetivo que el fisicalismo no alcanza.

El fisicalismo es una especie de monismo, opuesto a la distinción del dualista entre dos tipos de sustancia: la materia y la mente. En cuanto tal, desciende del materialismo: la concepción de que todo es materia (por ejemplo, la concepción de que no existe nada más que colecciones de átomos en el vacío) opuesta a, digamos, el dualismo cartesiano, que afirmaba que, así como existe la materia (sustancia extensa), así también existe la mente (sustancia pensante). Muchos fisicalistas consideran que su doctrina es una versión moderna del materialismo: defienden la hegemonía de la materia moderna contra los misterios de la sustancia mental y de la interacción mente-materia.

Pero el fisicalismo difiere significativamente de sus precedentes materialistas. En su forma mecanicista típica del siglo XVII, por ejemplo, el materialismo era una doctrina metafísica: trataba de limitar a priori la física mediante el requisito de que la materia fuese sólida, inerte, impenetrable, que se conservase, y que interactuase de forma determinista y sólo por contacto. Pero en su desarrollo posterior, la física ha mostrado que esta concepción de la materia era casi de todo punto incorrecta: la " materia" de la física moderna no es toda ella sólida, o inerte, o impenetrable, ni toda ella se conserva; e interactúa de forma indeterminista, y cabe argumentar que a veces lo hace a distancia. Enfrentados a estos descubrimientos, los descendientes modernos del materialismo han perdido, comprensiblemente, su coraje metafísico. Renunciando a limitar a priori la materia de la física, asumen ahora una actitud más servil: pretenden que el mundo empírico contiene exactamente aquello que una ciencia física verdadera y completa diría que contiene.

Pero esto plantea dos cuestiones. ¿Qué es la ciencia física: es decir, qué ciencias incluye este concepto? ¿Y qué le confiere esta autoridad ontológica? 
En otras palabras, ¿qué autoriza a ciertas ciencias a decirnos en sus propios términos lo que contiene el mundo, y por tanto a llevar el título honorífico del fisicalista, el título de "ciencias físicas"?

La "ciencia física" así construida incluye, ciertamente, la física propiamente dicha. La física es el paradigma (de ahí lo de "ciencia física"). Y la química, la biología molecular, y la neurofisiología son también indiscutiblemente ciencias físicas. Pero no lo son la psicología, la sociología y la economía. Cabe discutir los límites exactos de la ciencia física, pero a no ser que queden fuera de su ámbito algunas ciencias humanas, entre las cuales la psicología será nuestro ejemplar, el fisicalismo será vacuo en tanto que doctrina acerca de la mente.

¿Qué es lo que sitúa a la psicología más allá de los límites de lo físico? No es la metafísica a priori del materialismo del siglo XVII, dado que ésta ha sido refutada por la propia física. Tampoco es la negación materialista de la sustancia mental. La psicología también puede negar esa sustancia (y normalmente lo hace), pero aun así sigue sin contarse entre las ciencias físicas. La cuestión de si existe una "sustancia mental" además de la "sustancia física" es una cuestión irrelevante, puesto que esa contraposición de sustancias es en realidad una contraposición entre sus propiedades características: entre, digamos, pensar y ser extenso. Una sustancia meramente pensante no es una entidad físicamente respetable porque el pensamiento no es una propiedad físicamente respetable. ¿Pero por qué no? ¿Acaso no es la metafísica del materialismo lo que impide que la psicología empírica añada el pensamiento y otros fenómenos mentales al inventario de lo que existe, y que lo haga en sus propios términos, como lo hace la física?

A menudo se dice que las ciencias humanas han producido menores resultados que las ciencias reconocidas como ciencias físicas. Se afirma que sus leyes son escasas y poco consolidadas, y que sus teorías proliferan, y que predicen mucho menos que, digamos, la ley de la gravedad o las de la biología molecular. Quizás esta escasez de resultados proporciona una base epistémica para negar que la psicología y otras ciencias sean ciencias físicas, es decir, ciencias autorizadas para decirnos qué es lo que existe. Quizás simplemente no son lo bastante buenas.

Pero ésta no puede ser la razón por la que la psicología carece de la autoridad ontológica de la física, la química y esas otras ciencias. Como veremos, existen muchas leyes psicológicas bien establecidas. Y en cualquier caso, este argumento epistémico es erróneo porque está al revés. Quienes 
piensan que la psicología es sospechosa epistémicamente, lo hacen porque su objeto no es físico, y no viceversa. En otras palabras, tienen algún otro fundamento para suponer que la ciencia física excluye la psicología, un fundamento del cual supuestamente se sigue la inferioridad epistémica de la psicología. Y realmente es bastante obvio que este fundamento no es epistémico. Pues aceptar los resultados de la psicología no implica aceptarlos como resultados físicos: al contrario, cuanto más aceptan los fisicalistas tales resultados, tanto más creen que deben explicarlos (o eliminarlos mediante alguna explicación) en términos no psicológicos.

En realidad los límites de lo físico se establecen desde el principio. Se supone que hay algo en lo mental que priva a la psicología de la autoridad ontológica de la física y la química. ¿Pero qué es? ¿Qué impide que la psicología nos diga en sus propios términos qué clases de cosas y eventos mentales existen? Hay varias respuestas a esta pregunta, pero argumentaremos que ninguna de ellas justifica la exclusión prima facie de la psicología del ámbito de lo físico, exclusión que sería necesaria para hacer del fisicalismo una doctrina no vacía acerca de la mente.

\section{LA REDUCCIÓN A LA FÍSICA.}

A fin de evaluar las razones que tienen los fisicalistas para rechazar la psicología como una ciencia no física, y por tanto ontológicamente irrelevante, debemos preguntarnos qué es lo que les permite clasificar sus ciencias favoritas como ciencias físicas. ¿Qué hace que los fisicalistas incluyan entre las ciencias físicas no sólo las diversas ramas de la propia Física (mecánica, electromagnetismo, termodinámica, gravedad y física de partículas), sino también otras ciencias como la Química y la Biología molecular?

Una respuesta usual es que estas otras ciencias son físicas porque se reducen a la física, lo que para nuestros propósitos puede significar que una física dotada de principios puente adecuados (para conectar su vocabulario con el de esas otras ciencias) implicaría aproximaciones creíbles de todas las leyes establecidas por esas otras ciencias. ${ }^{3}$

De hecho, algunas teorías pertenecientes a otras ciencias físicas han sido reducidas a la física en este sentido, ${ }^{4}$ aunque de ningún modo lo han sido

${ }^{3}$ Cf. por ejemplo C. G. Hempel (1966, cap. 8).

${ }^{4}$ Cf. M. Friedman (1981). 
todas. Pero aquellos para quienes la reducción a la física es la piedra de toque de lo físico no proponen hacerlo en la práctica. Simplemente insisten en que puede hacerse "en principio". ¿Pero cuál es ese principio? No puede ser el fisicalismo. Estas ciencias no pueden ser reductibles en principio porque son físicas, si se supone que la reductibilidad en principio (REP) nos dice dónde están los límites de lo físico. Así pues, ¿qué otro principio nos dirá qué ciencias podrían ser reducidas "en principio" a la física?

Para contestar a esta pregunta, primero debemos preguntarnos a qué física se supone que se aplica el principio REP: ¿a la física actual, o a alguna hipotética física futura? Esta pregunta plantea un dilema. Aplicar el principio a la física actual implica que ninguna extensión futura de ésta sería a su vez física: implicaría que la física, el paradigma de la ciencia física, está ya completa. Pero nadie cree esto. Y si aplicamos el principio a alguna física futura sin especificar, no seremos capaces de decir qué ciencias son ciencias físicas mientras no sepamos cuáles de ellas quedarán cubiertas por esa física, que es precisamente lo que el principio debía decirnos. Incurrimos en un evidente círculo vicioso si aplicamos REP a la física futura para determinar lo que esa física ha de abarcar, si ha de abarcar todo lo que es físico. De manera que lo físico no puede definirse como aquello que es reducible en principio a la física, ni presente ni futura.

Creemos que el engañoso atractivo del principio REP se basa realmente en otros dos prejuicios. Uno es el viejo sueño de la "unidad de la ciencia", el sueño de ser capaces de derivar todas las leyes científicas a partir de un "gran esquema cada vez más adecuado". Pero no vemos ninguna razón ni para creer en ese gran esquema, ni para considerarlo como un objetivo a realizar. Incluso el mundo de las ciencias reconocidas como ciencias físicas contiene un vasto número de muy diferentes tipos de entidades, propiedades y hechos. Esta es la razón por la que, para estudiar esas entidades, propiedades y hechos, se necesitan tantas ciencias diferentes y que emplean métodos tan distintos. Nadie puede pensar que la astrofísica y la genética vayan a unificarse ni siquiera en sus métodos, excepto bajo las descripciones más abstractas de la metodología científica. Y en sus contenidos, no muestran más unidad que la de una yuxtaposición. No hay nada malo en esto, pero entonces ¿por qué no puede la psicología aportar otros elementos a esa yuxtaposición?

${ }^{5}$ H. Feigl (1963/1981, p. 315). 
Pero incluso si fuese creíble alguna versión de la tesis de la "unidad de la ciencia", eso tampoco permitiría que el principio REP definiese lo físico, pues ni siquiera la Física propiamente dicha está unificada. Quizás lo esté algún día; pero incluso si no llega a estarlo, los fisicalistas admitirán los fenómenos gravitatorios, cuánticos y electromagnéticos como fenómenos físicos que han de ser identificados y descritos en sus propios términos por ciencias físicas independientes. $\mathrm{Y}$ otro tanto sucederá con las ciencias de los fenómenos químicos, biológicos y neurofisiológicos. Por tanto, ¿por qué no aplicar lo mismo a la psicología, la ciencia de los fenómenos mentales?

La otra fuente del atractivo del principio REP es la idea de que en las cosas no hay realmente nada más que las partículas más pequeñas de las que están compuestas. Llamaremos a esto la tesis de la "microreducción", o para abreviar: tesis "MR". ${ }^{6}$ Esta idea es muy persistente. Tomemos las dos mesas de Eddington: su mesa común, con extensión, color y permanencia, frente a su mesa "científica", que no es nada más que una miríada de partículas minúsculas en el espacio vacío. La mesa cuya existencia me asegura "la física moderna mediante cuidadosas pruebas y una lógica despiadada ... es la única que existe realmente". ${ }^{7}$ O más recientemente, la afirmación de McGinn de que la ciencia nos dice que las cosas son muy distintas de lo que parecen. Según este autor, la física nos dice que la mesa que parece tan sólida a la vista y al tacto está realmente llena de agujeros. ${ }^{8}$

Pues bien, es verdad que el estudio de las entidades más pequeñas ha sido tradicionalmente denominado "Física": por una antigua costumbre, los departamentos de Física han acaparado ese mercado particular. Y esto hace que el principio MR afirme que el mundo empírico es físico, dado que únicamente consta de sus partículas más pequeñas. Retornamos a la doctrina de los átomos en el vacío (o al menos, en el campo), que se considera que son físicos simplemente porque son microscópicos.

El hecho de que, por una mera convención, la Física incluya el estudio de lo muy pequeño implica trivialmente que todo lo que tiene una extensión en el espacio, o bien es físico o bien tiene algunas partes físicas; y para algunos, esta verdad trivial es todo lo que significa el fisicalismo. ${ }^{9}$ Pero para que el

${ }^{6}$ G. Schlesinger (1963, cap. 2).

${ }^{7}$ A. S. Eddington (1929, pp. xi-xiv).

${ }^{8}$ C. McGinn (1983, cap. 7).

${ }^{9}$ G. P. Hellman y F. W. Thompson (1905). 
fisicalismo así definido no sea un concepto vacío, hay que asumir que estas cosas pequeñísimas son todo lo que existe. Ahora bien, ¿qué razón hay para pensar esto? ¿Por qué deberíamos suponer que la existencia de partículas subatómicas requiere la no existencia de los átomos, las moléculas, las mesas, los árboles, o las raquetas de tenis, los higos o los restaurantes de comida rápida - o los animales o las personas dotadas de una mente?

Quienes proponen el principio MR pueden, por supuesto, distinguir nuestra no existencia de la inexistencia de, digamos, los unicornios. Hay hechos indudables que al menos en apariencia son hechos acerca de nosotros, mientras que aparentemente no existen tales hechos acerca de los unicornios. Y por supuesto, dado que la propia física también estudia cosas muy grandes (galaxias, cuásares, etc.), MR también está obligado a decirnos por qué los hechos acerca de estas cosas, reconocidas como físicas, son diferentes de los hechos acerca de los unicornios, por un lado, y de los hechos acerca de las partículas subatómicas por otro lado. Lo que MR dice realmente es que todos estos hechos (acerca de las galaxias, las mentes, y todo lo demás) se reducen a hechos acerca de sus partes subatómicas. De manera que esas partes son todo lo que existe, quizás porque no necesitamos cuantificar ninguna otra cosa a fin de establecer todos esos hechos, y pensamos, con Quine, que no deberíamos multiplicar las entidades más allá de lo que sea cuantificacionalmente necesario. ${ }^{10}$

Pero esta apelación a la reducción muestra que el propio principio MR requiere una forma fuerte de ese mismo principio REP que se supone que MR debe apoyar. Y no puede tenerla. Pues a no ser que las ciencias que se ocupan de objetos relativamente grandes, incluida la psicología, se reduzcan a la microfísica, aun tendremos que cuantificar entidades descritas en los términos de esas ciencias. Pero en realidad, como vamos a mostrar ahora, ni siquiera la física de lo relativamente grande se reduce a la microfísica. De manera que, incluso si todas las ciencias fuesen reductibles en principio a la física, esto no implicaría que las partículas más pequeñas son todo lo que existe: MR sería falso incluso si el principio REP fuese verdadero. De modo que el principio REP no puede utilizarse para apoyar MR.

Lo que sí es verdad es que los hechos acerca de las partes a menudo explican los hechos acerca de los todos. Considerada como una tesis acerca de la explicación, MR es a menudo una buena hipótesis de trabajo. Pero no

${ }^{10}$ W. V. O. Quine (1953). 
siempre se verifica, ni siquiera en microfísica. Si, por ejemplo, consideramos que la descripción que ofrece la mecánica cuántica de un conjunto cuántico es completa (como hacen las interpretaciones ortodoxas), el principio de superposición implica que sus propiedades no serán una función únicamente de sus constituyentes aislados y de las relaciones entre ellos. La física cuántica ortodoxa no es microreductiva. Y una parte de la física es claramente macroreductiva: por ejemplo, el principio de Mach que hace depender la masa inercial incluso de las micropartículas de cómo está distribuida la materia por todo el universo. Somos conscientes, por supuesto, de que el principio de Mach y la teoría cuántica ortodoxa son controvertidas, y que una física futura bien puede abandonar ambas cosas. Pero no pueden ser abandonadas con el argumento de que entran en conflicto con un principio MR supuesto por la moderna microfísica, dado que, como se puede ver, la microfísica no supone nada parecido.

Y el principio MR ni siquiera se cumple siempre en la física más ordinaria. Normalmente es verdad que allí donde van las partes de algo, el todo debe ir también: que una muestra de gas debe ir allí donde vayan sus moléculas. Pero es igualmente cierto que sus moléculas deben ir allí donde va la muestra, pues si no lo hiciesen cesarían en el acto de ser sus moléculas. Y éste no es el único modo en el que las moléculas de un gas son gobernadas por éste en la misma medida en que éste es gobernado por ellas. Supongamos, por ejemplo, que el volumen de nuestra muestra gaseosa es súbitamente reducido a la mitad manteniendo constante la temperatura. Si se trata de un gas ideal, la ley de Boyle implica que cuando la presión remita su volumen volverá a duplicarse. Esa ley no dicta todo el comportamiento de las moléculas de la muestra durante todo el proceso, excepto que debe ser tal que eventualmente multiplique por dos la presión del conjunto. Esa parte del comportamiento de esas moléculas está determinada (y por tanto explicada) de un modo macroreductivo mediante una ley que gobierna la totalidad de la muestra.

Así pues, en tanto que principio explicativo, MR no siempre se cumple, ni siquiera en la física. Por consiguiente, su valor explicativo no puede servir para convertirlo en una tesis ontológica. A fortiori no puede apoyar el fisicalismo. Pero ni siquiera podría hacerlo si fuese verdadero, pues ninguna interpretación correcta de MR podría implicar que las entidades macroscópicas, y sus propiedades y relaciones, quedan refutadas por estar conectadas mediante leyes con propiedades y relaciones de sus partes más pequeñas. No pueden serlo. Pues si lo fuesen, tendrían que existir algunas entidades minúsculas, sin 
partes: es decir, un límite a la estructura de la materia a pequeña escala. Pero está claro que no es necesario que exista ese límite, de manera que la existencia de las partículas más pequeñas que conocemos actualmente no podría ser refutada por el descubrimiento de partículas aún menores en el interior de ellas. Pero entonces también los átomos deberán poder coexistir con sus partes subatómicas, las moléculas con sus átomos, y así sucesivamente, hasta llegar a las mesas, los árboles $-\mathrm{y}$ nosotros. La existencia de animales y personas, con sus propiedades y relaciones psicológicas y sociales, no puede ser negada simplemente porque se les atribuyan partes lo bastante pequeñas como para interesar a la microfísica.

El hecho de que la física comience por lo muy pequeño ha fomentado el mito de que es una ciencia universal en un sentido en el que otras (como la psicología) no lo son, siendo meramente "ciencias especiales". ${ }^{11} \mathrm{Ha}$ fomentado este mito porque hace que todo aquello que sea más grande que un punto tenga, por definición, propiedades que son físicas (o tenga partes lo bastante pequeñas como para tener esas propiedades). De forma parecida, todo lo que se mueve tiene propiedades físicas, tales como la masa inercial, simplemente porque la física incluye, por definición, la ciencia de la mecánica. Pero esto no convierte a estas ciencias en ciencias universales en el sentido de que abarquen todas las propiedades y relaciones de las cosas; ni las convierte en ciencias básicas, en el sentido de que otras ciencias tengan que reducirse a ellas. En cualquier sentido que sustentase una definición no vacía de lo físico, que es lo que necesita el fisicalismo, la mecánica y la microfísica no son más universales o básicas que la psicología. Son simplemente las ciencias especiales del movimiento y de lo muy pequeño.

En resumen, si los fenómenos de la psicología son menos aceptables ontológicamente que los de la física y la química, ello no puede deberse a que la psicología sea irreductible a la física presente o futura. La reductibilidad a la física, o a la microfísica, es un test desastroso para juzgar la autoridad ontológica de una ciencia: un test que ni siquiera un fisicalista puede aplicar consistentemente. Pues, como hemos visto, en la practica la reductibilidad ni es factible ni es relevante; y aquellos que afirman la reductibilidad "en principio", o bien incurren en una petición de principio, o bien apelan a principios tales como el de la unidad de la ciencia o el de la microreducción, principios que la propia física moderna rechaza.

${ }^{11}$ Cf. J. A. Fodor (1974). 


\section{CAUSACIÓN MENTAL E INTENCIONALIDAD}

¿De qué otro modo podría la ciencia física (es decir, la ciencia dotada de autoridad ontológica) ser definida de manera tal que excluya la psicología? Quizás mediante la causación, que muchos piensan que es esencialmente física. Quizás lo físico es simplemente lo causal, y lo que realmente significa el fisicalismo es que el mundo empírico comprende todas (y sólo) aquellas entidades, propiedades, relaciones y hechos que tienen causas o efectos. Esta definición subyace claramente a una formulación familiar del problema mentecuerpo: ¿cómo pueden los estados mentales tener efectos en un mundo físico? Esta cuestión no supondría un problema si no se asumiese que la causación es esencialmente no mental.

¿Pero por qué deberíamos asumir eso? Sin duda es obvio que existe bastante causación mental. Supongamos que ves a un amigo y esto es la causa de que lo saludes: ¿cómo sucede esto? Más o menos así: la luz reflejada por tu amigo llega a tu retina; los impulsos ascienden por tu nervio óptico; tu córtex estriado procesa la información portada por esos impulsos; formas (de algún modo) la creencia de que tu amigo está ahí; esto hace que formes la intención de saludar a tu amigo; esto hace que sucedan ciertas cosas en tu sistema motor; esas cosas causan que tu brazo se levante ... Esta cadena de causación parece involucrar igualmente hechos físicos y mentales. ¿Cómo puede entonces un fisicalismo definido por la causación excluir estas causas y efectos aparentemente mentales?

Ciertamente, no es nueva la idea de que la causación mental es difícilmente inteligible, y especialmente la causación que conecta lo mental con lo no mental, dado que ambos parecen ser muy diferentes. Pero ¿por qué debería impresionar esto a cualquiera que haya aprendido de Hume que la causación nunca "es inteligible", que es siempre una cuestión de hecho, no de razón? Nada en los análisis humeanos o en otros análisis modernos de la causación obliga a las causas a ser como sus efectos; y nada en ellos impide que las causas y los efectos sean mentales.

Consideremos la exigencia de que las causas y efectos concretos se localicen en el espacio y en el tiempo, de tal modo que puedan ser contiguos (o, si es necesario, densos o continuos) y de este modo unos puedan preceder a otros. Las sensaciones concretas e incluso los pensamientos concretos pueden 
ciertamente ser localizados con precisión suficiente para cumplir esa exigencia (dado que localizar un pensamiento concreto no supone localizar su ilocalizable contenido abstracto en mayor medida de lo que localizar un objeto rojo supone localizar el color abstracto "rojo"). Nada en lo mental impide que los pensamientos, sentimientos y sensaciones concretas de las personas se encuentren en el lugar y en el momento en que se encuentran esas personas, a fin de estar allí donde puedan tener las causas y efectos inmediatos y por tanto contiguos, mentales y no mentales, que claramente parecen tener.

Otras condiciones usualmente exigidas a la causación se cumplen también en la misma medida por las causas y efectos mentales y por los no mentales: por ejemplo, la exigencia de que las causas sean en cada caso suficientes para sus efectos, o necesarias, o ambas cosas; o la exigencia de que las causas hagan que los efectos sean más probables de lo que lo serían sin esas causas; o la exigencia de que las relaciones causales instancien leyes. Es difícil ver por qué cualquiera de estas condiciones debería suponer un obstáculo a la existencia de causas y efectos mentales.

Si existe un problema con la causación mental, dicho problema reside en la intencionalidad, la capacidad de la mente de representar aspectos del mundo. Y de hecho, a menudo se supone que la intencionalidad impide que los fenómenos mentales sean, como tales, fenómenos físicos. Así se expresa Fodor:

Supongo que más tarde o más temprano los físicos completarán el catálogo que han estado compilando de las propiedades últimas e irreductibles de las cosas. Cuando lo hagan, cosas tales como spin, charm y carga quizás aparezcan en su lista. Pero está claro que la propiedad de ser acerca de algo [aboutness] no estará en ese catálogo. Simplemente, la intencionalidad no arraiga tan abajo. ${ }^{12}$

Pero en la sección anterior ya hemos discutido las pretensiones de la física de proporcionar todas las "propiedades últimas e irreductibles de las cosas". Y nadie a quien hayan impresionado nuestros argumentos pensará que es importante que la intencionalidad arraigue menos "profundamente" en este sentido que el spin y la carga. Pues muchas propiedades no mentales (por ejemplo, químicas y biológicas) tampoco figurarán en el catálogo del físico. Y si esto no las impugna ni impugna tampoco a las entidades a las que caracterizan,

${ }^{12}$ J. A. Fodor (1987, p. 97). 
¿por qué debería impugnar la intencionalidad o las entidades a las que ésta caracteriza?

Pero muchos filósofos seguirían estando de acuerdo con el siguiente comentario de Fodor según el cual "la motivación más profunda para el irrealismo intencional deriva de una intuición ontológica: la intuición de que no hay lugar para las categorías intencionales en una visión fisicalista del mundo". De este modo, Field escribe: "cualquier materialista que crea en la realidad de las creencias y los deseos (...) debe mostrar que las relaciones en cuestión no son irreductiblemente mentales." "13 Si esta intuición fuese correcta, y existiesen razones independientes para aceptar su "visión fisicalista del mundo", realmente tendríamos razón al negar la realidad de la intencionalidad, y por tanto de buena parte de lo mental, si es que no todo. Pero, como veremos, esta intuición es errónea.

¿Cuál se supone que es el problema de la intencionalidad? Los estados intencionales tienen típicamente tres rasgos distintivos: (i) parecen ser afectados por objetos o eventos distantes, y causar acciones que involucran tales objetos o eventos; (ii) su adscripción crea contextos no extensionales (proposiciones cuyo valor de verdad puede alterarse cuando los nombres o las descripciones que aparecen en ellas se reemplazan por otras que se aplican a las mismas cosas); y (iii) pueden referirse a objetos o eventos que no existen. Supongamos por ejemplo que (i) lees algo acerca de Santa Fe que hace que quieras ir allí, lo cual causa que subas a un avión y lo hagas. Pero (ii) no quieres ir a la ciudad más hermosa de Nuevo México, que es Santa Fe, porque no sabes que lo es. Y (iii) podrías haber querido ir a Santa Fe incluso si, como Eldorado, no existiese.

El desafío que plantean (i) y (iii) es explicar cómo puede Santa Fe causar que actúes como lo haces estando tan lejos, y sin necesitar siquiera existir. Nadie cree que una ciudad pueda tener tales efectos directamente a tales distancias -especialmente si no necesita existir. Tu acción puede estar causada directamente por alguna propiedad intrínseca que tengas realmente, no por tus relaciones con objetos distantes y que pueden ser inexistentes, como Santa Fe, o con objetos abstractos como la proposición, que puede ser falsa, de que esa ciudad está en Nuevo México.

Pero esto no significa que los poderes causales de los pensamientos y otros estados mentales concretos no puedan depender de sus contenidos:

${ }^{13}$ H. H. Field (1978, p. 78). 
pueden depender de éstos. Lo único que significa es que deben hacerlo indirectamente, a través de una representación mental, es decir, a través de alguna propiedad intrínseca no relacional del estado mental (o de su poseedor). Un pensamiento concreto debe tener alguna propiedad intrínseca de ese tipo, correlacionada de algún modo con su contenido, para darle a este sus causas y efectos oportunos. Una instancia de esa propiedad es, si se nos permite decirlo así, el sustituto causal local [local causal surrogate] de ese contenido.

Pero estas propiedades intrínsecas podrían ser también mentales. Podrían ser sensaciones, o imágenes o modelos visuales o de otro tipo mental -no necesariamente conscientes, dicho sea de paso. ${ }^{14} \mathrm{E}$ incluso si la estructura composicional del pensamiento requiere que estas instancias concretas formen una estructura compleja correspondiente ("sintáctica"), podría estar formada por imágenes (como las figuras de Shepard compuestas de imágenes de cubos). ${ }^{15}$ De manera que el problema que los pensamientos suponen para la causación no es que sean mentales y la causación no lo es. El problema es que la causación depende directamente sólo de propiedades intrínsecas, mientras que los poderes causales de los pensamientos concretos dependen de sus contenidos, que no son intrínsecos. ${ }^{16}$ Esto muestra, en efecto, que estos contenidos necesitan sustitutos causales: pero no porque sean mentales, dado que los sustitutos pueden ser también mentales.

Además, la necesidad de sustitutos causales en modo alguno pertenece únicamente al campo de la psicología. Tales sustitutos se necesitan en todo el ámbito de las ciencias físicas. Por ejemplo, una función estándar de los campos físicos consiste en proporcionar sustitutos causales locales para aquello que, de otro modo, sería una acción inmediata a distancia. Pero nadie piensa que aceptar los campos gravitatorios newtonianos signifique negar el status físico o la existencia de la gravedad newtoniana que median esos campos.

En otras partes de la física y la química, los sustitutos causales son necesarios también para realizar algo que de otro modo sería una causación hacia atrás. Ya señalamos antes que la ley de Boyle hace que la eventual presión de una muestra de gas ideal se duplique después de que su volumen sea súbitamente reducido a la mitad manteniendo constante la temperatura. Pero el equilibrio de presión concreto, $P$, no puede afectar directamente a los procesos

${ }^{14}$ Cf. P. N. Johnson-Laird (1983); R. N. Shepard y L. A. Cooper (1982).

15 Shepard y Cooper (1982, cap. 3).

${ }^{16}$ Uno de nosotros lo ha mostrado detalladamente en otra parte: T. M. Crane (1989). 
carentes de equilibrio que conducen a él, dado que esto requeriría una causación hacia atrás. Además, $P$, como Santa Fe, ni siquiera necesita existir. El volumen de la muestra puede alterarse de nuevo antes de alcanzar el equilibrio: pero esto no puede afectar a su comportamiento anterior. De manera que el futuro estado $P$ necesita un sustituto causal en el presente para hacer que la muestra se dirija hacia $P$, exactamente como Santa Fe necesita un sustituto causal en ti para hacer que te dirijas hacia allí.

Y lo mismo que sucede en este caso, sucede en todos los sistemas que tienden a un equilibrio estable: desde el simple péndulo a las reacciones químicas y biológicas de todo tipo. Por ejemplo, en termodinámica química los potenciales de Gibb son sustitutos causales para los equilibrios a los que tienden los sistemas químicos; ${ }^{17}$ equilibrios cuya existencia y estatus físico no quedan impugnados por tales sustitutos causales, como tampoco los mecanismos de los campos gravitatorios newtonianos impugnan la gravedad, ni la cinética de los gases refuta la ley de Boyle ni muestra que reducir a la mitad el volumen de una muestra de gas no sea realmente la causa de que su presión se duplique. Por consiguiente, ¿por qué las explicaciones fisiológicas o psicológicas del modo en que los contenidos de los pensamientos concretos producen sus efectos los contradicen, o contradicen las explicaciones causales que dan de nuestras acciones?

Con esto terminamos el análisis de los problemas supuestamente planteados por (i) y (iii) para la autoridad ontológica de la psicología intencional. ¿Y qué sucede con (ii), la no extensionalidad de las adscripciones de estados mentales intencionales? Este rasgo tampoco excluye lo mental de lo físico, dado que la no extensionalidad también aparece en la física. ${ }^{18}$ Esto se debe a que las leyes implican condicionales no extensionales. Supongamos, por ejemplo, que $\mathrm{H}$ y $\mathrm{K}$ son los genes que nos proporcionan corazones y riñones. El hecho de que todos tengamos ambas cosas no hace que la proposición "todo el que tuviera el gen $\mathrm{H}$ tendría un corazón" implique "todo el que tuviera el gen K tendría un corazón" o "todo el que tuviera el gen $\mathrm{H}$ tendría un riñón".

Las leyes probabilísticas de la microfísica moderna no pueden ser extensionales también por otra razón: porque " $p(\ldots)=n$ " no es extensional. Pues si lo fuese, " $a$ es el $F$ " y la verdad necesaria " $p(a$ es $a)=1$ " implicarían " $p(a$

${ }^{17}$ K. G. Denbigh (1955, p. 76).

18 B. Enc (1982). 
es el $F)=1$ ", lo que claramente no es el caso en ninguna concepción de la probabilidad (tomemos como ejemplo " $F$ "= "próximo Primer Ministro").

La no extensionalidad de la probabilidad explica, de paso, la no extensionalidad de muchos ejemplos causales singulares de los contextos “... porque...", incluso en física. Esto se debe a que la causación da probabilidades a los efectos, si bien sólo subjetivas. Las explicaciones probabilistas de la causación explicitan esto, que está implícito incluso en las explicaciones deterministas. ${ }^{19}$ Los efectos de las causas suficientes, por ejemplo, tienen probabilidad 1; y los efectos de las causas necesarias tendrían en su ausencia la probabilidad 0. De manera que "E porque..." debe ser no extensional, puesto que, si bien que $a$ sea el $F$ puede dar a " $E$ " una probabilidad contingente, el que $a$ sea $a$ no puede hacerlo. $\mathrm{Y}$ “ “... porque $C$ ” tampoco puede ser extensional, debido a sus implicaciones contrafácticas: la probabilidad de una verdad necesaria como " $a$ es $a$ " no puede depender de $C$, incluso si depende de ella la probabilidad de una proposición verdadera como " $a$ es el $F$ ".

Estas y otras razones nos convencen, pese a lo que opinan Davidson y otros, de que incluso en la física la causación singular nunca depende de, y generalmente no es, una relación causal extensional entre particulares. ${ }^{20}$ Pero si los contextos causales pueden ser no extensionales de todas formas, pueden contener perfectamente bien contextos no extensionales como "cree ...", " quiere ...", "teme ...", etc.: como en " $b$ teme que $a$ sea el $F$ porque $a$ se lo dijo", o " $b$ hace $D$ porque quiere que $a$ sea el $F$ y cree que sólo lo será si ella hace $D$ ". Así pues, no vemos razones para negar la causación que estas proposiciones obviamente expresan, o para suponer que dicha causación debe basarse en, o reducirse a, alguna relación causal extensional $-\mathrm{y}$ menos aún a una relación no mental que relaciona particulares no mentales.

En resumen, todos los rasgos supuestamente problemáticos de los estados intencionales son tan endémicos a la física, y en particular a la causación no mental, como lo son a la psicología. Por consiguiente, la noción de causación no servirá para definir las ciencias físicas (y por tanto aquellas que tienen autoridad ontológica) de modo tal que quede excluida la psicología. Definir lo físico como lo causal no hará del fisicalismo una doctrina no vacía acerca de la mente.

19 Por ejemplo D. H. Mellor (1988); J. L. Mackie, (1965/1975).

${ }^{20}$ D. Davidson, (1967/1980; D. H. Mellor (1987). 


\section{LEYES PSICOLÓGICAS Y PSICOFÍSICAS.}

Para que la cuestión del fisicalismo sea una cuestión seria, debe haber una distinción de principio entre lo mental y lo físico que explique por qué las ciencias no mentales tienen una autoridad ontológica de la que carece la psicología. Hemos visto que ni la causación ni la reductibilidad a la física pueden proporcionar dicha distinción. Pero quizás puedan hacerlo las leyes. Cabe argumentar que la autoridad ontológica de la ciencia se basa en las leyes que descubre, leyes que nos dicen qué clases de cosas existen y qué propiedades y relaciones las distinguen. Pero muchos coinciden con Davidson en que lo mental es " anómalo": en que estrictamente hablando no existen las leyes psicológicas o psicofísicas. ${ }^{21} \mathrm{Si}$ esto fuese cierto, la psicología no añadiría nada a nuestra ontología de las clases no mentales, con sus propiedades y relaciones distintivas no mentales.

Pero ¿por qué deberíamos negar que, por ejemplo, "todos los hombres son mortales" (una generalización psicofísica verdadera que pone un límite a la consciencia [sentience] de los miembros de nuestra especie) es una ley? Hay algunas malas razones para negar que lo sea, razones que no consideraremos en detalle. Una de ellas es la idea de que las leyes son necesariamente verdaderas, algo que no serán nunca las generalizaciones acerca de lo mental. Así, según McGinn, que los términos mentales figuren en leyes significa que "las generalizaciones universales que contienen términos mentales [son] metafísicamente necesarias". 22 Pero, argumenta este autor (influido por el conocido argumento de Kripke contra la teoría de la identidad), ${ }^{23}$ ninguna generalización no analítica necesariamente verdadera enlaza términos mentales con términos no mentales, ni con otros términos mentales.

No lo hacen, pero ninguna generalización de este tipo enlaza tampoco los términos de la física unos con otros. Las leyes de la física no son metafísicamente necesarias. Concedemos a Davidson que las leyes deben estar "apoyadas en sus ejemplos" y "apoyar afirmaciones contrafácticas y subjuntivas" ("si $x$ fuese $F$ sería G"). ${ }^{24}$ Pero "todos los hombres son mortales" puede cumplir claramente estas condiciones sin ser una verdad necesaria: el hecho de que algo no viviría para siempre si fuese humano no significa que no

${ }^{21}$ D. Davidson, "Mental Events" (1970/1980).

${ }^{22}$ C. McGinn (1980, p. 187).

${ }^{23}$ S. Kripke (1972).

${ }^{24}$ D. Davidson (1970/1980, p. 217). 
pudiese hacerlo, como tampoco "si fuésemos allí, iríamos en autobús" significa que no pudiésemos ir en tren. Por consiguiente, tampoco el hecho de que las leyes de la física cumplan estas condiciones muestra que sean verdades necesarias; y uno de nosotros ha argumentado en otra parte que no lo son. ${ }^{25}$ De manera que, si para que los términos cuenten como términos físicos tienen que figurar en verdades necesarias no analíticas, los términos de la física no contarían como tales, independientemente de lo que suceda con los términos de la psicología.

La ley según la cual todos los Fs son $G$ sólo implica que cualquier cosa que fuese $F$ sería $G$, no que deba serlo. (Y la ley probabilística según la cual todos los Fs tienen una probabilidad $p$ de ser $G$, donde $0<p<1$, ni siquiera implica eso, aun si se trata de una verdad necesaria.) La mera posibilidad de que existan excepciones a las generalizaciones psicológicas y psicofísicas no hace, pues, que dejen de ser leyes. E incluso si lo hiciese, incluso si las leyes tuviesen que ser tanto necesarias como deterministas, ¿cómo se podría mostrar, sin incurrir en petición de principio, que una generalización verdadera podría realmente tener excepciones? No podría hacerse simplemente imaginando esas excepciones. Todos podemos imaginar que la luz viaja en el vacío más rápidamente que a su actual velocidad $c$. Esto no prueba que realmente pueda viajar más rápidamente, y menos aún que la constancia de $c$ no sea una ley. Y algo similar sucede en psicología. Cualquiera puede imaginar que el agua de mar sabe a oporto: aun así, puede existir una ley según la cual nadie que tenga nuestras papilas gustativas podría nunca saborear algo así.

Otra mala razón para negar la existencia de leyes psicofísicas es la llamada "realización variable" de los estados mentales: el hecho de que "la gama de estados físicos aptos para realizar un estado mental dado puede ser indefinidamente variada". ${ }^{26}$ Esto no impide que las generalizaciones psicofísicas sean leyes. Pues si lo impidiese, apenas habría leyes tampoco en la física. Estados tales como las masas, los volúmenes y las temperaturas se realizan de un modo incluso más diverso que los estados mentales: podemos tener un gramo o un litro de casi cualquier cosa a cualquiera de una innumerable infinidad de temperaturas. ${ }^{27}$ Así que, si la realización variable no

${ }^{25}$ Cf. D. H. Mellor (1980); D. M. Armstrong (1983).

${ }^{26}$ C. McGinn (1978, p. 197).

${ }^{27}$ Cf. M. Wilson (1985, p. 235). 
excluye las leyes en mecánica y termodinámica, difícilmente puede excluirlas en psicología.

Tampoco deberíamos dejarnos impresionar por el hecho de que quienes reflexionan desde su sillón sean incapaces de excogitar generalizaciones psicológicas o psicofísicas. La física y la química no pueden excogitarse a priori, y no vemos ninguna razón por la que la psicología tendría que poder serlo. Descubrir la psicofísica del gusto, o de la visión, ${ }^{28}$ o la psicología inconsciente de la inferencia, ${ }^{29}$ puede exigir tantas y tan complejas teorías y experimentos como desentrañar, digamos, la bioquímica de la reproducción.

En particular, el fracaso de Stich a la hora de excogitar leyes en las que figuren estados mentales intencionales no significa que tales leyes no existan. ${ }^{30}$ La explicación obvia de nuestra incapacidad para establecer tales leyes en formas simples y carentes de excepciones es que nuestra psicología intencional es demasiado compleja y (probablemente) probabilística. Pero también lo es la meteorología de los huracanes, y la mecánica cuántica de las macromoléculas. Por esas razones, no podemos establecer sus leyes en formas simples y carentes de excepciones. Nadie infiere de esto que no existen tales leyes; y esa inferencia no es mejor en el caso de la psicología.

El propio Davidson no emplea estos argumentos contra la existencia de leyes psicológicas. Su argumento es el siguiente:

(1) No hay leyes psicofísicas estrictas.

(2) Las causas y los efectos singulares deben instanciar leyes estrictas.

(3) Lo mental no es un sistema "comprehensivo cerrado", al ser afectado por lo no mental, que sí forma un sistema tal.

Pero por (1) estos efectos mentales no pueden instanciar leyes psicofísicas estrictas. Por consiguiente,

(4) "No existen en absoluto leyes estrictas sobre la base de las cuales podamos predecir y explicar fenómenos mentales". ${ }^{31}$

${ }^{28}$ D. Marr (1982).

${ }^{29}$ P. N. Johnson-Laird (1983).

30 S. Stich (1985, cap. 7).

${ }^{31}$ D. Davidson (1970/1980, pp. 224-225). 
Este argumento falla en cada uno de sus pasos. La premisa (1) es falsa, y no sólo porque "todos los hombres son mortales" es una ley. Hay muchas más leyes de este tipo, que conectan sensaciones (dolores, olores, sabores, y sensaciones visuales, auditivas y táctiles) con rasgos no mentales de quienes tienen esas sensaciones. Deben existir tales leyes, puesto que industrias enteras dependen de ellas. Pensemos en las leyes que deben subyacer a la producción y empleo fiables de anestésicos, aromas, narcóticos, edulcorantes, pinturas y luces coloreadas, altavoces y cojines). $\mathrm{Y}$ si las leyes newtonianas del movimiento bastan para que añadamos las masas y las fuerzas a nuestra ontología física, estas leyes deben bastar para añadir a dicha ontología las clases de sensaciones que figuran en ellas.

Pero incluso si no existiesen tales leyes psicofísicas, no por eso se socavaría la autoridad ontológica de la psicología. Incluso si ninguna ley conectase lo mental con lo no mental, la psicología podría tener sus propias leyes que definiesen su propia ontología mental, en pie de igualdad con la ontología de, digamos, la química. Pues como vimos en la sección 2 , la autoridad ontológica de la química no depende de que pueda reducirse a la física a través de leyes físico-químicas. Por consiguiente, tampoco la autoridad ontológica de la psicología depende de que existan leyes psicofísicas. ${ }^{32}$

Pero como hemos visto, de hecho nuestras sensaciones están sujetas a leyes psicofísicas, que bastan por sí mismas para refutar la afirmación de Davidson de que no "puede haber leyes estrictas que conecten lo mental con lo [no mental]", 33 dado que tales leyes bien pueden ser "estrictas" (es decir, deterministas). Es verdad que Davidson está más interesado en los estados intencionales que en las sensaciones; pero nuestra refutación sigue siendo válida, dado que las sensaciones son indiscutiblemente mentales (como el propio Davidson admite). ${ }^{34}$

Así pues, (1) es falsa. Pero también lo es (2): las causas y los efectos sólo necesitan instanciar leyes probabilísticas. ${ }^{35}$ Pero entonces, ¿no podemos hacer que (2) sea verdadera - y reforzar (4) - eliminando en todo el argumento la condición de que las leyes sean "estrictas"? En efecto, podemos

\footnotetext{
32 Cf. W. Lycan (1981).

${ }^{33}$ D. Davidson (1970/1980, p. 212).

${ }^{34}$ Davidson (1970/1980, p. 211); cf. también Davidson (1985, p. 246).

${ }^{35}$ Cf. por ejemplo P. Suppes, (1985); D. H. Mellor (1988).
} 
hacerlo, y deberíamos hacerlo: pero esto no ayuda a Davidson, puesto que lo único que se consigue con esto es hacer (1) aún más increíble.

Admitir las leyes probabilísticas tampoco sirve de nada para salvar la premisa (3). Pues tanto si la causación necesita leyes estrictas o leyes meramente probabilísticas, lo no mental sigue sin formar un "sistema comprehensivo cerrado" en mayor medida en que lo hace lo mental.

Pues ¿qué significa "un sistema comprehensivo cerrado"? Para Davidson, significa un sistema de leyes "homonómicas", que "pueden tener la esperanza de ser precisas, explícitas y tan carentes de excepciones como sea posible" sólo porque extraen sus conceptos "de una teoría comprehensiva cerrada". Las ciencias no mentales pueden proporcionar una teoría tal, afirma Davidson; pero la psicología no puede hacerlo. Sus generalizaciones son irremediablemente "heteronómicas": es decir, pueden "darnos razones para creer que hay alguna ley precisa funcionando, pero una ley que sólo puede establecerse si nos desplazamos a un vocabulario diferente". ${ }^{36}$

Pero esta distinción no nos sirve, dado que la propia física está llena de leyes heteronómicas. Consideremos la mecánica newtoniana, que define los conceptos newtonianos de fuerza y masa diciendo cómo se combinan para causar la aceleración. Las leyes del movimiento que hacen esto no forman una teoría cerrada. De hecho, sin alguna ley ulterior que relacione la fuerza con otros conceptos, no forman ninguna teoría contrastable en absoluto. En la teoría que aparece en los Principia de Newton, esa ley ulterior es la ley de la inversa del cuadrado de la gravedad. Pero tampoco esa teoría es una teoría cerrada. Hay muchas otras clases de fuerza: eléctrica, magnética, viscosa, etc. De manera que, como ley de fuerza neta, la ley de la gravedad de Newton es tan irremediablemente heteronómica como las leyes de la psicología: sólo puede convertirse en una ley sin excepciones mediante condiciones que invocan conceptos ajenos de electricidad, etcétera. ${ }^{37} \mathrm{Y}$ algo similar sucede con todas las otras leyes de fuerza. Todas ellas son verdaderas sólo en tanto que leyes de clases de fuerzas: gravitacional, eléctrica, etc., las cuales se combinan en fuerzas netas mediante adición vectorial. La teoría de la mecánica newtoniana es simplemente la conjunción de todas esas leyes con las leyes newtonianas del movimiento, por muy diversos que sean sus otros conceptos.

${ }^{36}$ D. Davidson (1970/1980, p. 219).

${ }^{37}$ Cf. N. Cartwright (1983, cap. 3). 
Por supuesto, la mecánica newtoniana ha sido reemplazada, pero no porque fuese sólo una conjunción: pues una conjunción, tal como vimos en la sección 2 , tiene toda la unidad que una ciencia necesita. De modo que nuestra "teoría comprehensiva cerrada" puede ser también una simple conjunción: la conjunción de todas las teorías y leyes científicas verdaderas. Pero entonces, decir, como hace Davidson, que las ciencias no mentales pueden proporcionar esta conjunción por sí mismas, es simplemente negar la existencia de leyes psicológicas; lo cual incurre en una petición de principio y además se ve refutado por las leyes que sabemos que conectan las sensaciones con sus causas no mentales.

Así pues, el argumento de Davidson (1)-(4) fracasa completamente en el intento de mostrar que no hay leyes psicofísicas o psicológicas. Pero esto no refuta su tesis de que no hay leyes que conecten los estados mentales intencionales. Y para esa tesis Davidson da un argumento especial, que se basa principalmente en dos ideas conectadas: el "holismo" de lo intencional, y el "ideal constitutivo de la racionalidad".

El holismo de los estados mentales intencionales significa que dichos estados son conceptualmente interdependientes, a diferencia de las sensaciones. La creencia de que $P$, por ejemplo, debe inhibir la creencia de que no- $P$, y también el deseo de que $P$ (la gente no quiere lo que piensa que ya tiene). Por otra parte, ni la creencia ni el deseo pueden causar por sí solos la acción. Para hacerlo deben combinarse, y diferentes combinaciones pueden causar la misma acción: por ejemplo, puedo decir " $P$ " o bien porque lo creo y quiero decir la verdad, o bien porque no lo creo y quiero mentir. $\mathrm{Y}$ no hay duda de que tales relaciones familiares entre creencias, deseos y acciones los definen en parte, y por tanto impiden que cualesquiera leyes que los incluyan sean plenamente independientes.

Pero estos hechos no pueden impedir que dichas leyes existan, puesto que también ellos tienen paralelos newtonianos. La fuerza $(f)$ y la masa $(m)$ newtonianas también son conceptualmente interdependientes, al ser parcialmente definidas mediante la relación $f=m a$, que impide que las leyes que las incorporan sean independientes entre sí. Y también esta relación requiere que las fuerzas y las masas se combinen para producir sus efectos (las aceleraciones), y permite que muchas combinaciones causen el mismo efecto. De manera que no podemos inferir una fuerza $f$ o una masa $m$ a partir de la aceleración $a$ que causan, como tampoco podemos inferir una creencia o un deseo a partir de la acción que causan. En una palabra, el holismo no es 
suficiente por sí solo para distinguir lo intencional de lo no mental de tal modo que lo mental se muestre como anómalo, como por otra parte Davidson admite. ${ }^{38}$

¿Y qué podemos decir del "ideal constitutivo de la racionalidad" de Davidson? Se trata de la idea de que las relaciones entre creencias, deseos y acciones mencionadas más arriba en parte definen o constituyen (de ahí lo de "constitutivo") lo que es ser racional. Por ejemplo, el hecho de que la creencia de que $P$ generalmente inhibe la creencia de que no- $P$ es uno de los truismos holísticos que ayudan a definir la racionalidad: es racional no tener pares de creencias obviamente contradictorias. La racionalidad es un ideal porque los que piensan pueden ser más o menos racionales: puede que no tengan la totalidad de sus estados intencionales en estas relaciones "racionales".

Puede que todo esto sea verdadero, pero una vez más no puede descartar las leyes psicológicas, dado que todo ello tiene también un paralelo newtoniano. De hecho, todo lo que según Davidson es peculiar de "nuestro uso de los conceptos de creencia, deseo y demás" tiene un paralelo newtoniano. He aquí lo que dice:

A medida que se acumulen las pruebas [de aceleraciones inducidas por la gravedad, la electricidad, etc.], deberemos estar preparados para ajustar nuestra teoría [de las fuerzas y masas implicadas] a la luz de consideraciones de fuerza lógica [que satisfagan las leyes de Newton]: el ideal constitutivo de racionalidad [las leyes de Newton] controla parcialmente cada fase de la evolución de lo que debe ser una teoría en evolución. Una elección arbitraria del esquema de traducción [de las aceleraciones a las fuerzas] excluiría ese oportuno ajuste de la teoría: dicho de otro modo, una correcta elección arbitraria de un manual de instrucciones sería una elección de un manual aceptable a la luz de todas las pruebas posibles, y ésta es una elección que no podemos hacer. ${ }^{39}$

Hemos puesto en cursiva las dos analogías en cuestión. En primer lugar la racionalidad, que para muchos es una noción normativa que limita, por ejemplo, lo que uno debe creer. Bueno, quizás lo sea, pero la racionalidad de una creencia puede seguir siendo un hecho acerca de ella, por ejemplo, algo que hace probable que sea verdadera; y el ideal constitutivo simplemente exigiría que las creencias estén relacionadas de tal modo entre sí, y con sus causas

${ }^{38}$ D. Davidson (1970/1980, p. 221); D. Davidson (1985, p. 248).

${ }^{39}$ Davidson (1970/1980, p. 223). 
perceptivas, que en condiciones normales la mayoría de ellas sea verdadera. Y lejos de impedir que haya leyes que conecten los contenidos de nuestras creencias con nuestro entorno y con la operación no mental de nuestros sentidos, esto requiere positivamente que existan tales leyes (aunque sean sólo probabilísticas).

En segundo lugar tenemos la afirmación de que ninguna evidencia empírica puede permitirnos escoger un esquema de traducción correcto: es decir, un esquema que infiera correctamente creencias, deseos, etc., a partir de sus causas perceptivas y de sus efectos conductuales. Pero si esto ha de marcar una diferencia con la mecánica, no puede significar que ninguna evidencia empírica puede implicar la teoría correcta. Esto es manifiesto en la mecánica newtoniana incluso si las fuerzas son observables, dado que toda adscripción de una masa en cualquier tiempo $t$ implica una infinidad de aceleraciones netas bajo diferentes fuerzas netas en $t$, ninguna de las cuales implica ninguna otra, y de las cuales sólo una puede ser real. ¿Cómo podrían los estados mentales intencionales estar más infradeterminados que esto por las pruebas que tenemos de ellos?

Sin embargo, Davidson cree que la "indeterminación de la traducción" de Quine muestra que deben estarlo. ${ }^{40}$ Afirma que la anomalía de lo mental "se remonta" a la "función central de la traducción" y su indeterminación. Pues si no existe una traducción determinada de las proposiciones, no existe una afirmación correcta de lo que significan. De manera que, dado que sus significados son los contenidos de las creencias que expresarían, tampoco existe una afirmación correcta de éstos: es decir, las creencias (y a fortiori otros estados mentales intencionales) no tienen contenidos determinados. Los contenidos, como los significados de las proposiciones, no están simplemente infradeterminados por las evidencias empíricas: sencillamente no existen. Y si no existen, ciertamente no puede haber ejemplos de ellos que figuren en leyes.

Pero nosotros negamos la indeterminación de la traducción, por razones ya familiares. ${ }^{41}$ Como ya hemos visto, y como muchos otros han señalado, ${ }^{42}$ la indeterminación de la traducción no puede inferirse de la infradeterminación de las teorías por sus pruebas, pues en tal caso ni siquiera la mecánica newtoniana tendría leyes. Ni tampoco puede inferirse de "la

40 Quine (1960, cap. 2); Davidson (1970/1980, p. 222).

${ }^{41}$ Cf. por ejemplo Kirk (1986, parte III).

42 Por ejemplo Chomsky (1969). 
pretensión de Quine de que las teorías de la traducción están (...) infradeterminadas incluso por la totalidad de las verdades expresables en los términos de la física" ${ }^{43}$ sin incurrir en una petición de principio, dado que por hipótesis las leyes psicológicas y psicofísicas no pueden expresarse en esos términos. Basar la indeterminación de la traducción en esa pretensión es basarla en algo que, como vimos en la sección 2 , es una versión insostenible (la versión de la reductibilidad a la física) de ese mismo fisicalismo que se pretende apoyar mediante dicha pretensión.

No conocemos ninguna otra razón para negar a priori la existencia de leyes que impliquen estados mentales intencionales. Pero ¿no podría la totalidad de todas las teorías no mentales verdaderas ser de becho tan comprehensiva y cerrada como para excluir las leyes psicológicas y psicofísicas? No vemos cómo. Ninguna cantidad de física, por grande que sea, podría impedir que los estados mentales instancien otras leyes también. Por supuesto, esas otras leyes deben ser consistentes con la física, pero sólo porque todas las verdades deben ser consistentes entre sí. Este truismo no da prioridad a la física, cuyas leyes deben ser igualmente consistentes con las de la psicología.

Pero quizás esta lectura de (3) puede parecer más plausible como una tesis acerca de la causación, más que como una tesis acerca de las leyes: y el propio Davidson sugiere que así es como él interpreta (3), cuando dice que "hay demasiadas cosas que afectan a lo mental que no son ellas mismas una parte sistemática de lo mental". ${ }^{4}$

Supongamos que la física formase un sistema causal comprehensivo, de tal modo que las leyes de la física hiciesen que cada uno de tus estados cerebrales o movimientos corporales $b_{2}$ en cualquier tiempo $t_{2}$ estuviesen determinados por tus estados cerebrales en un tiempo anterior $t_{1}$ (y por el input no mental entre $t_{1}$ y $\left.t_{2}\right)$. ¿Cómo podrían tus estados mentales entre $t_{1}$ y $t_{2}$ afectar también a $b_{2}$ sin violar estas leyes deterministas?

Consideremos ahora un caso paralelo. Supongamos que las leyes de Kepler hiciesen que la posición orbital $p_{2}$ de la Tierra en $t_{2}$ estuviese determinada por su posición $p_{1}$ en $t_{1}$ (además de su velocidad en ese momento, $\mathrm{y}$ del input desde el espacio entre $t_{1}$ y $t_{2}$ ). Igualmente podemos preguntarnos, ¿cómo podrían las posiciones de la Tierra entre $t_{1}$ y $t_{2}$ afectar también a $p_{2} \sin$ violar las leyes de Kepler? Sin embargo deben afectar a $p_{2}$ si $p_{1}$ lo hace, pues $p_{1}$

${ }^{43}$ Kirk (1986, p. 136).

${ }^{44}$ Davidson (1970/1980, p. 224). 
sucede entre $t_{2}$ y otras posiciones anteriores $p_{0}$ que, dadas las leyes de Kepler, también determinan $p_{2}$. No hay nada especial en $t_{1}$.

La solución a este rompecabezas reside en el condicional contrafáctico (C) implicado en esta afirmación causal: si $p_{1}$ hubiese sido diferente, también lo habría sido $p_{2}$, pero no $p_{0}$. En otras palabras, lo que viola las leyes de Kepler es sólo el antecedente contrafáctico de $(\mathrm{C})$. El propio $(\mathrm{C})$ no las viola, y por tanto tampoco lo hace la afirmación causal que lo implica: de hecho son las leyes de Kepler las que hacen que (C) sea verdadero, y por tanto que lo sea la afirmación causal.

Algo similar sucede en nuestro caso original. Nuestros estados mentales, intencionales y de otro tipo, podrían afectar a nuestros estados cerebrales y movimientos corporales (y lo harían), incluso si las leyes de la física hiciesen que todos ellos estuviesen determinados también por estados cerebrales anteriores. La afirmación de que un sistema limitado de este modo por leyes no mentales debe ser un sistema cerrado, en el sentido de no poder ser afectado por sus estados mentales, simplemente no se sigue de lo anterior y no es verdadera.

\section{SUPERVENIENCIA.}

Hemos visto que ni las leyes ni la causación privan a la psicología de la autoridad ontológica de las ciencias no mentales. Pero esto todavía deja en pie una interpretación no vacía del fisicalismo. El último refugio del fisicalista moderno es la superveniencia: la tesis de que no hay cambio o diferencia sin un cambio o diferencia no mental. Dos cosas nunca cambiarán o diferirán en modo alguno si no cambian o difieren de algún modo no mental. Lo físico excluye lo mental al ser aquello en lo que sobreviene [supervenes] todo lo demás, incluido lo mental.

La superveniencia es más fuerte que la afirmación trivial de que todo lo que se extiende en el espacio tiene partes físicas, pero más débil que el reduccionismo, dado que no afirma nada acerca de cuál diferencia no mental acompañará a cualquier diferencia mental: no implica la existencia de ninguna ley psicofísica. Pero debe ser más fuerte de lo que hemos indicado hasta aquí. Pues, dada la multitud de propiedades no mentales susceptibles de cambio que posee cualquier cosa (incluida su localización espaciotemporal), de hecho todas las cosas que cambian o difieren mentalmente deben cambiar o diferir en algún 
aspecto no mental. De manera que la superveniencia, si es seria, debe significar algo más que eso. La gama relevante de aspectos no mentales debe ser restringida (excluyendo al menos la ubicación espaciotemporal), y la afirmación debe ser al menos subjuntiva ("dos cosas nunca diferirían..."), y cabría argumentar que fuese incluso más fuerte ("dos cosas nunca podrían diferir...").

Sin embargo, para plantar cara a la superveniencia la interpretaremos del modo más débil posible: en su forma subjuntiva, y limitando lo menos posible los aspectos no mentales relevantes. Incluso así, no vemos razones para creer en ella. Las pruebas a su favor no pueden ser empíricas, dado que es extremadamente reducida, en el mejor de los casos, la perspectiva de encontrar alguna vez dos cosas lo bastante complejas como para tener propiedades psicológicas y que sean de tipo idéntico en todos los aspectos no mentales razonables. El único argumento remotamente plausible a favor de la superveniencia es uno que apela al principio causal mencionado en la sección 3, según el cual no existe la acción inmediata a distancia. Esto significa, como ya vimos, que las creencias y otros estados mentales intencionales concretos necesitan propiedades intrínsecas que actúen como sustitutos causales de sus contenidos. Y si todas estas propiedades son no mentales, y las sensaciones están igualmente determinadas por sus causas no mentales, entonces la superveniencia parece ser una conclusión correcta.

Pero no lo es. En primer lugar, como ya hemos observado, las propiedades intrínsecas que actúan como sustitutos causales de los contenidos de las creencias y otros estados intencionales concretos pueden perfectamente ser mentales. $\mathrm{Y}$ en segundo lugar, tanto si son mentales como si no lo son, las creencias no sobrevendrán en ellos. Dos seres pensantes pueden fácilmente tener las mismas propiedades intrínsecas y aun así tener creencias diferentes. Esta es una obvia moraleja de las historias de Putnam acerca de la "tierra gemela": ${ }^{45}$ el contenido de la creencia de tu doble en la tierra gemela de que el agua es húmeda difiere de tu creencia, puesto que el agua de tu doble es XYZ y la tuya es $\mathrm{H}_{2} \mathrm{O}$. Y otro tanto sucede con las creencias indexicales. Si el contenido de "eso es un olmo" incluye el árbol al que miras mientras piensas esta proposición, ese contenido será diferente en dos personas que miran árboles diferentes, incluso si ambas tienen las mismas propiedades intrínsecas.

El partidario de la superveniencia puede replicar que esto sólo muestra que los pensamientos no sobrevienen en las propiedades intrínsecas de quienes

45 Putnam (1975). Dicha conclusión es extraída expresamente por Burge (1986). 
los piensan. Pueden sobrevenir en las restantes relaciones no mentales de los pensadores con otras cosas (por ejemplo, en las relaciones espacio temporales), y en las propiedades no mentales de esas cosas (ser un olmo, $\mathrm{o}_{2} \mathrm{O}$ ). Pero esto tampoco es verdad, como podemos ver si consideramos cómo quienes piensan cometen errores. Supongamos por ejemplo que tú y tu gemelo intrínsecamente idéntico percibís ahora el mismo olmo, pero esto hace que tú pienses que es un olmo, mientras que hace que él o ella piensen que es un roble. Tenemos aquí las mismas propiedades intrínsecas, las mismas relaciones, las mismas propiedades de la cosa acerca de la que se piensa; pero tenemos pensamientos diferentes.

De nuevo, el partidario de la superveniencia podría replicar que en tal caso siempre habría alguna diferencia no mental relevante: si no en los ojos, sí en la apariencia del árbol desde los diferentes puntos de vista. Pero nosotros dudamos de esto. Tú y tu gemelo podéis diferir sólo en aspectos mentales: por ejemplo, en las creencias acerca del aspecto de los olmos -creencias que no necesitan sobrevenir en algo presente o no mental, sino simplemente en los diferentes efectos mentales que los árboles han tenido sobre vosotros en el pasado. $\mathrm{Y}$ no vemos ninguna razón para pensar que tales efectos deben sobrevenir en diferencias no mentales pasadas, a no ser que incurramos en una petición de principio.

Por otro lado, tus experiencias pasadas y las de tu gemelo tienen efectos presentes: pueden hacer que pienses "eso es un olmo", y que tu gemelo piense "eso es un roble". Y estando a cierta distancia temporal, no pueden tener esos efectos inmediatamente: sus efectos deben estar mediados por algunas propiedades intrínsecas presentes que se encuentran en ti y en tu gemelo. Así pues, ¿quizás tus pensamientos deben sobrevenir en tus propiedades intrínsecas, después de todo?

No es así. Pues no sólo sucede, como hemos visto, que estas propiedades mediadoras pueden ser ellas mismas mentales, sino que, incluso si no lo son, no necesitan ser diferentes sólo porque sus efectos mentales lo sean. Después de todo, la causación no necesita ser determinista, y la física moderna nos dice que a menudo no lo es. Así que tenemos razones sobradas para esperar algún tipo de indeterminismo en los procesos causales de nuestra percepción, nuestros razonamientos, y nuestra acción; y éste es un modo en el que estos procesos pueden salir mal y hacernos cometer errores. Pero cuando la causación es indeterminista, las causas y los efectos no sobrevendrán unos en otros. En una palabra, la física moderna nos da razones para negar la 
superveniencia de los contenidos de nuestros pensamientos concretos en incluso la más extensa lista de nuestras restantes propiedades y relaciones intrínsecas y extrínsecas.

Y lo que vale para los pensamientos, vale para las sensaciones. Que tengan causas o efectos no mentales no hará que sean supervenientes. $\mathrm{Al}$ contrario, si la causación relevante es indeterminista en algún sentido, las sensaciones no pueden sobrevenir en sus causas no mentales.

No obstante, los partidarios de la superveniencia pueden replicar que la causación, a diferencia de la superveniencia, requiere tiempo -y estamos de acuerdo con eso. ${ }^{46}$ Las causas siempre preceden a sus efectos, mientras que los pensamientos y las sensaciones concretas sólo se supone que sobrevienen en instancias simultáneas de propiedades no mentales. Así pues, mostrar que no sobrevienen en sus causas no mentales anteriores no refuta directamente esa afirmación.

Pero la refuta indirectamente. Supongamos que una propiedad no mental $P$ causa una propiedad mental $M$ de forma indeterminista. (Digamos, por ejemplo, que la probabilidad de que uno sea $M$ en $t_{2}$ es 0.9 si uno ha sido $P$ (en $t_{1}$ ), y 0.1 si no lo ha sido). Supongamos ahora que en $t_{1}$ muchas personas comparten todas sus propiedades intrínsecas no mentales, incluida $P$. En $t_{2}$, por consiguiente, la mayoría de ellos, pero no todos, serán $M$ : es decir, algunos pares de personas, semejantes átomo a átomo en $t_{1}$, serán diferentes en $t_{2}$ en este aspecto mental.

Sean $a$ y $b$ uno de sus pares: en $t_{2}$, $a$ es $M$ y $b$ no lo es. ¿Qué sucede con las propiedades intrínsecas no mentales de $a$ y $b$ en $t_{2}$ ? Bueno, todas ellas pueden estar determinadas por el estado no mental compartido por $a$ y $b$ en $t_{1}$. Pero si es así, entonces también todas ellas serán compartidas, y $M$ tampoco sobrevendrá en ellas. Pero $M$ no sobrevendrá en ellas de todas formas. Pues incluso si algunas leyes relevantes de la física son indeterministas, de tal modo que el estado de $a$ y $b$ en $t_{1}$ no hace que compartan todas sus propiedades intrínsecas no mentales en $t_{2}$, ello no impedirá que lo hagan. Al contrario, dado un número suficiente de as y bs, con certeza algunos diferirán mentalmente en $t_{2}$ sin diferir en ningún otro sentido.

En otras palabras, la física indeterminista moderna debe predecir que algunos pares de personas, iguales átomo a átomo en todos los aspectos no mentales, serán diferentes en algunos aspectos simultáneos mentales; y lo serán

${ }^{46}$ D. H. Mellor (1981, caps. 9-10). 
precisamente porque las propiedades implicadas están relacionadas causalmente. En resumen, la física moderna sugiere que incluso la forma seria más débil de superveniencia, que a su vez es la forma más débil de fisicalismo no vacío, es falsa. Y sin duda los fisicalistas no pueden esperar que un fisicalismo que ha sido falsado por la física sea verificado por alguna otra cosa.

\section{FIN.}

Hemos argumentado que ninguna definición defendible del fisicalismo privará a la psicología del estatus ontológico de las ciencias no mentales. El fisicalismo no es verdadero en ningún sentido no vacío. Pero esto no significa que queramos alentar un renacimiento del dualismo cartesiano. Al contrario, nuestros argumentos implican que no existen una división entre lo mental y lo no mental que baste siquiera para plantear el fisicalismo como un problema serio, y no digamos como una respuesta seria a ese problema. El fisicalismo es la respuesta equivocada a un problema esencialmente trivial. Así que no puede ayudar a los filósofos de la mente a responder las cuestiones serias acerca de la mente y, sobre todo, acerca de la intencionalidad: qué habilita a algunas partes del mundo (nosotros) a pensar acerca de otras partes, incluidas otras personas (y por supuesto nosotros mismos). Y es bastante obvio que ni el dualismo ni el fisicalismo tienen nada que decir acerca de esas cuestiones. El dualista ni siquiera intenta explicar la intencionalidad: simplemente la da por supuesta, estipulando su existencia. Y decir que las mentes son enteramente físicas no ayuda a explicar cómo algunas cosas físicas pueden pensar, como tampoco decir que toda la carne es hierba ayuda a explicar la diferencia entre carnívoros y vegetarianos. Por consiguiente, éste debería ser realmente el último artículo sobre el tema del fisicalismo. Pero nos tememos que no lo será. ${ }^{47}$

\footnotetext{
${ }^{47} \mathrm{El}$ material de este artículo en parte procede de la tesis doctoral de uno de los autores (T. M. Crane, op. cit.), y en parte del material presentado por el otro autor en reuniones de varias instituciones: British Society for the Philosophy of Science, Oxford University Philosophical Society, Cambridge University PPE Workshop, así como en seminarios de diversas universidades: University College London, University of Sussex, University of California at Irvine, University of Wisconsin at Madison, NorthWestern University y el History and Philosophy of Science Department de Cambridge. Agradecemos los útiles comentarios y críticas que nos hicieron muchas personas en esas ocasiones y en muchas conversaciones y cartas privadas.
} 


\author{
Tim Crane \\ University of Cambridge \\ tc102@cam.ac.uk \\ D.H. Mellor \\ University of Cambridge \\ bugh.mellor@me.com
}

\title{
BIBLIOGRAFÍA
}

Armstrong, D. M. (1983), What is a Law of Nature?, Cambridge: Cambridge University Press.

Burge, T. (1986), "Individualism and Psychology", Philosophical Review.

CARTwright, N. (1983), How the Laws of Physics Lie, Oxford: Clarendon Press.

Crane, T. (1982), The Content and Causation of Thought, Cambridge University Ph.D. Dissertation.

ChOmsky, N. (1969), "Quine's Empirical Assumptions", en D. Davidson y J. Hintikka (eds.), Words and Objections, Dordrecht: Reidel.

Davidson, D. (1967/1980), "Causal Relations", en D. Davidson, Essays on Actions and Events, Oxford, Clarendon Press.

Davidson, D. (1970/1980), "Mental Events", en D. Davidson, Essays on Actions and Events, Oxford, Clarendon Press.

DAvidson, D. (1985), "Replies to Essays", en B. Vermazen y M. Hintikka (eds.), Essays on Davidson: Actions and Events, Oxford: Clarendon Press.

Denbigh, K. G. (1955), The Principles of Chemical Equilibrium, Cambridge: Cambridge University Press.

Eddington, A. S. (1929), The Nature of the Physical World, Cambridge: Cambridge University Press.

ENC, B. (1982) "Intentional States of Mechanical Systems", Mind.

FIELD, H. H. (1978), "Mental Representation", Erkenntnis.

FIELD, H. H. (1980), Sciencie without numbers, Oxford: Blackwell, 1980.

Feigl, H. (1963/1981), "Physicalism, Unity of Science and the Foundations of Psychology", en H. Feigl Inquiries \& Provocations, R. Cohen (ed.), Dordrecht: Reidel.

FODOR, J. A. (1974), "Special Sciences", Synthèse.

FODOR, J. A. (1987), Psychosemantics: the Problem of Meaning in the Philosophy of Mind, Cambridge, Mass.: MIT Press.

Friedman, M. (1981), “Theoretical Explanation”, en R. Healey (ed.), Reduction, Time and Reality, Cambridge: Cambridge University Press. 
Hellman, G. P. y Thompson, F. W. (1905), "Physicalism: Ontology, Determination and Reduction", Journal of Philosophy.

Hempel, C. G., (1966), Philosophy of Natural Science, Englewood Cliffs, NJ: PrenticeHall.

JOHnson-LAird, P. N. (1983), Mental Models, Cambridge: Cambridge University Press.

KIRK, R. (1986), Translation Determined, Oxford: Clarendon Press.

KRIPKE, S. (1972), "Naming and Necessity", en D. Davidson y G. Harman (eds.), Semantics of Natural Languages, Dordrecht, Reidel.

LONGUET-Higgins, C. (1072), "The Failure of Reductionism", en C. LonguetHiggins et al., The Nature of Mind, Edinburgh: Edinburgh University Press.

LYCAN, W. (1981), "Psychological Laws", Philosophical Topics.

MACKIE, J. L. (1965/1975), "Causes and Conditions", en E. Sosa (ed.), Causation and Conditionals, Oxford: Oxford University Press.

MARr, D. (1982), Vision, San Francisco, Freeman.

MCGINN, C. (1978), "Mental States, Natural Kinds, and Psychophysical Laws I", Proceedings of the Aristotelian Society Supplementary Volume.

MCGINN, C. (1980), "Philosophical Materialism", Synthèse.

MCGINN, C. (1983), The Subjective View, Oxford: Claredon Press.

MELlOR, D. H. (1980), "Necessities and Universals in Natural Laws", en D. H. Mellor (ed.), Science, Belief \& Behaviour, Cambridge: Cambridge University Press.

Mellor, D. H. (1981), Real Time, Cambridge: Cambridge University Press.

MELLOR, D. H. (1987), “The Singularly Affecting Facts of Causation”, en D. Pettit et al. (eds.), Metaphysics and Morality, Oxford: Blackwell.

Mellor, D. H. (1988), "On Raising the Chances of Effects", en J. H. Fetzer (ed.), Probability and Causality, Dordrecht: Reidel.

Putnam, H. (1975), "The Meaning of «Meaning»", en Mind, Language and Reality, Cambridge: Cambridge University Press.

Quine, W. V. O. (1953), “On What There Is", en W. V. O. Quine, From a Logical Point of View, Harvard University Press.

Quine, W. V. O. (1960), Word and Object, Cambridge, Mass.: MIT Press.

SHEPARD R. N. y COOPER, L. A. (1982), Mental Images and Their Transformations, Cambridge, Mass.: MIT Press.

Schlesinger, G. (1963), Method in the Physical Sciences, London: Routledge \& Kegan Paul.

STICH, S. (1985), From Folk Psychology ti Cognitive Science, Cambridge, Mass.: MIT Press.

Stroud, B, (1987), "The Physical World", Proceedings of the Aristotelian Society.

SupPES, P. (1985), "Davidson's Views of Psychology as a Science”, en B. Vermazen y M. Hintikka (eds.), Essays on Davidson: Actions and Events, Oxford: Clarendon Press. 
Wilson, M. (1985), "What is this Thing Called «Pain»? - the Philosophy of Science Behind the Contemporary Debate", Pacific Philosophical Quarterly. 